\title{
Scan direction influences explicit but not implicit perception of a goalkeeper's position
}

\author{
Benjamin Noël ${ }^{1}$ - John van der Kamp ${ }^{2,3} \cdot$ Rich Masters $^{3,4} \cdot$ Daniel Memmert ${ }^{1}$
}

Published online: 29 August 2016

(C) The Psychonomic Society, Inc. 2016

\begin{abstract}
In soccer penalty kicking, it has been demonstrated that systematic biases in a penalty taker's explicit perception of the goalkeeper's position do not always show up in decisions about the side to which to kick. To scrutinize whether this off-center effect is a function of dissociations between explicit and implicit perception of the goalkeeper's position, we examined to what degree visual scan direction affects explicit as well as implicit perception of goalkeeper position. To this end, participants were presented with pictures of a goalkeeper who stood at different (marginal) distances to the right or left of goal center. To manipulate scan direction, participants fixated the right, middle, or left of the scene at the beginning of each trial. They were instructed only to kick the ball if they perceived the goalkeeper to be standing in the center of the goal. Results showed that scan direction systematically influenced explicit perception of goalkeeper position (i.e., the decision to kick). Yet, if participants decided to kick (and thus believed that the goalkeeper stood in the true center), then the kicks were more often directed to the side with more space (i.e., $64.1 \%$ ) irrespective of scan direction. These findings provide further evidence that the off-center effect arises from
\end{abstract}

Benjamin Noël

b.noel@dshs-koeln.de

1 Institute of Cognitive and Team/Racket Sport Research, German Sport University Cologne, Cologne, Germany

2 MOVE Research Institute Amsterdam, Faculty of Behavioral and Movement Sciences, VU University Amsterdam,

Amsterdam, Netherlands

3 Institute of Human Performance, University of Hong Kong, Hong Kong, China

4 Department of Sport and Leisure Studies, University of Waikato, Hamilton, New Zealand dissociations between explicit and implicit perception of goalkeeper position, with the former but not the latter being susceptible to attentional asymmetries.

Keywords Landmark discrimination task $\cdot$ Implicit perception · Attentional asymmetries · Off-center effect . Penalty kicking

Accurate perception of space is key in sports. However, asymmetries in spatial perception have been shown recently in golf putting (Roberts \& Turnbull, 2010), Australian football goal kicking (Nicholls, Loetscher, \& Rademacher, 2010), beach volleyball (Noël, Hüttermann, van der Kamp, \& Memmert, 2016), and association soccer penalty kicking (Noël, van der Kamp, Weigelt, \& Memmert, 2015). Soccer players preparing to take a penalty kick, for instance, err in identifying the true center of the goal. When asked to position the goalkeeper in the center of the goal before taking a penalty kick, experienced soccer players systematically placed the goalkeeper to the right of center (Noël, et al., 2015). This is analogous to asymmetries in spatial perception observed in line bisection tasks (Jewell \& McCourt, 2000). Intriguingly, although the soccer players typically failed to identify the true center, their subsequent kick tended to be directed to the side of the goal with more space. The players were not instructed to kick to the side with more space and genuinely believed that the goalkeeper was standing in the goal's center. This phenomenon is known as the off-center effect (Masters, van der Kamp, \& Jackson, 2007).

A soccer penalty kick, however, more closely approximates the landmark discrimination task (Milner, Brechmann, \& Pagliarini, 1992) than the line bisection task. In the landmark discrimination task participants judge whether a preset mark bisects a horizontal line at its center (i.e., whether the 
two halves of the line are of equal length). In a soccer penalty kick, players do not guide the goalkeeper to the goal's center (as in the line bisection task), but rather they judge whether the goalkeeper is standing in the goal's center (as in the landmark discrimination task), and then kick. Masters et al. (2007, Exp. 3 ) adopted this procedure in a penalty kick task; the goalkeeper stood at different positions relative to the goal's center and players only kicked when they felt sure that the goalkeeper stood in the true center. Consequently, players were unaware that the goalkeeper was standing off-center when they kicked. Nevertheless, they more often directed the ball to the side with more space, suggesting that decisions to which side to shoot relied on implicit perception of the goalkeeper's true position (or differences in the magnitude of space to goalkeeper's right and left).

In the current study, we aimed to provide further evidence that the off-center effect arises from dissociations between explicit and implicit perception of the goalkeeper's position relative to the goal center. To this end, we assessed whether or not conditions that affect explicit perception of goalkeeper position extend to presumably implicit decisions about to which side to kick. In fact, line bisection and landmark discrimination studies have identified many factors that bias the direction and magnitude of explicit perception of a line's center (Jewell \& McCourt, 2000). One such factor is the direction from which the observer searches or scans the line or scene. For a horizontal line in far space, scanning the line from left to right, biases perception of the center to the right of the line's true center more than when the line is scanned in the opposite direction from right to left (Varnava, McCarthy, \& Beaumont, 2002). We tested whether scan direction also influences a penalty taker's perception of the goalkeeper's position in the goal and whether scan direction impacts decision-making for the subsequent kicking action. To this end, participants viewed images of a goalkeeper standing in the goal's center or marginally to the left or right of the center (throughout the current article displacements are always described from the penalty taker's perspective). Participants were instructed to penalty kick only if they perceived the goalkeeper to be in the true center of the goal. Before viewing each image, participants were required to fixate a cross on the screen. The cross was located where the right or left goal post, or the goalkeeper appeared to induce scanning towards the goalkeeper from the left, the right, or centrally. Consistent with Varnava et al. (2002), we expected that the perceived center of the goal would change as a function of scan direction, with goalkeepers positioned to the right of center perceived to be standing in the center of the goal when scanning initiated from the left side compared with the right side and vice versa. Hence, the decision to kick (or not to kick) was expected to be a function of scan direction. In cases where the goalkeeper was consciously perceived to be standing in the true center, penalty kicks were expected. If the concomitant decision to which side to kick did not rely on explicit perception of goalkeeper position but instead involves presumably more accurate implicit processes (Noël, van der Kamp et al., 2015), then kick direction should have been independent of scan direction but be biased to the side with more space. Alternatively, if the decision to which side to kick relied on explicit perception, then the side with more space should have been chosen more often when participants started scanning from the side with more space compared with the side with less space (i.e., kicks to the side that was predominantly scanned, see Noël \& van der Kamp, 2012; van der Kamp, 2011; Wood \& Wilson 2010).

\section{Methods}

\section{Participants}

Twenty students (13 males, 2 left-footed, 24.8 years of age, $\mathrm{SD}=2.9)$ took part in the experiment. Participants reported normal or corrected to normal vision. The experiment was performed in accordance with the World Medical Association Helsinki Declaration 2008 and approved by the local ethics committee. Sample size requirements were calculated using G*Power 3.1 (Faul, Erdfelder, Buchner, \& Lang, 2009), indicating that a sample size of 18 participants would result in sufficient power $(0.80)$ to detect significant differences $(\alpha$-level $=0.05, f=0.2)$.

\section{Apparatus}

E-Prime 2.0 software was used to present the stimuli. Images of a goalkeeper standing on the goal line were projected onto a reinforced screen (i.e., a screen protected by a perspex pane) in the laboratory. The goal's dimensions and the distance between the penalty mark and the screen were scaled to $23 \%$ of dimensions prescribed by the FIFA regulations. A soft football (size 5) was used. To verify that participants complied with the instructions regarding their gaze behaviors, SMI Eye Tracking Glasses were used.

\section{Procedure and design}

After providing informed consent, participants were tested on their capability to make four kicks to designated target areas $(15 \times 15 \mathrm{~cm})$ projected on the left and right side of the screen. Successful participants were fitted with the eye tracker and completed 270 experimental trials. In each trial, a goalkeeper was shown standing on the goal line at different distances to the left or right of the center of the goal. At the start of each trial, a fixation cross was shown for 2 seconds at the location where the left or right post or the goalkeeper would subsequently appear. The goalkeeper was positioned in the goal's 
center or displaced to either side of the center. Based on previous research (Weigelt \& Memmert, 2012; Weigelt, Memmert, \& Schack, 2012) marginal displacements (i.e., difficult to detect explicitly) were $0.92,1.15$, and $1.38 \mathrm{~cm}$ (i.e., corresponding to 4,5 , and $6 \mathrm{~cm}$ in a soccer goal) and an easily detectable displacement was $6.9 \mathrm{~cm}(30 \mathrm{~cm})$. Each fixation cross location and displacement combination was presented ten times in random order.

A trial started when participants stood one step behind the ball at the penalty mark and signaled they were ready. Participants were instructed to fixate the cross until the goal and the goalkeeper appeared after which they were free to orient their gaze. This induced scanning across the scene from left to right or right to left toward the goalkeeper or gaze that remained oriented centrally. Participants also were told that when the goal and the goalkeeper appeared they were to kick and try to score a goal only if they perceived the goalkeeper to be standing in the exact center of the goal. Additionally, participants were instructed to refrain from kicking if they perceived the goalkeeper to be standing off-center. Participants had to indicate verbally whether their decision was to kick (and immediately do so) or not to kick.

\section{Analysis}

Videos were analyzed offline to confirm that participants were gazing at the location of the fixation cross when the stimulus appeared. Only trials in which this was the case were included in the analyses (i.e., $>96 \%$ of trials). Analyses of gaze started when the picture appeared until participants indicated they had decided (i.e., typically just before they shifted gaze toward the ball if they performed a kick). The percentage of trials in which participants decided to kick and the percentage of kicks to the side with more space served as dependent variables. To examine the influence of goalkeeper displacement and location of fixation cross, the percentage of trials in which participants kicked was submitted to a 3 (fixation cross location: left, right, center) $\times 9$ (displacement: 0 , and $0.92,1.15,1.38$, and $6.9 \mathrm{~cm}$ to the left and right) ANOVA with repeated measures on both factors. Bonferroni corrected pairwise comparisons were carried out to identify differences between means. A similar repeated measures ANOVA with fixation cross location and displacement (i.e., without the $0 \mathrm{~cm}$ condition because the two sides are equally large) was planned to assess differences in the percentage of kicks to the side with more space. Finally, a series of separate one-sample $t$ tests (test value $50 \%$, Bonferroni corrected) assessed whether or not the side with more space was selected in the majority of the trials for each displacement. Either partial eta-squared $\left(\eta_{\mathrm{p}}^{2}\right)$ or Cohen's $d$ were calculated to determine the proportion of total variability attributable to each (combination of) factor(s), or the standardized differences between means respectively.

\section{Results}

All volunteers succeeded in hitting the designated areas, suggesting that they were capable of kicking the ball relatively accurately. In the vast majority of trials (i.e., $>0.98 \%$ ), the initial fixation on the cross at the left or right side of the goal was followed by a gaze shift toward the goalkeeper, inducing predominant left to right or right to left scanning directions depending on the location of the fixation cross. Once the goalkeeper was fixated, and also in trials with a central fixation cross, gaze remained stationary until participants indicated whether or not they would kick. There were no additional gaze shifts toward either side of the goal; typically, however, a decision to kick was followed by a gaze shift toward the ball.

Figure 1 illustrates the percentage of trials in which the participants decided to kick. It shows that participants' decision to kick at smaller displacements (i.e., because they perceived the goalkeeper to stand in the center of the goal) was systematically biased by scan and displacement directions. The ANOVA revealed a significant main effect of

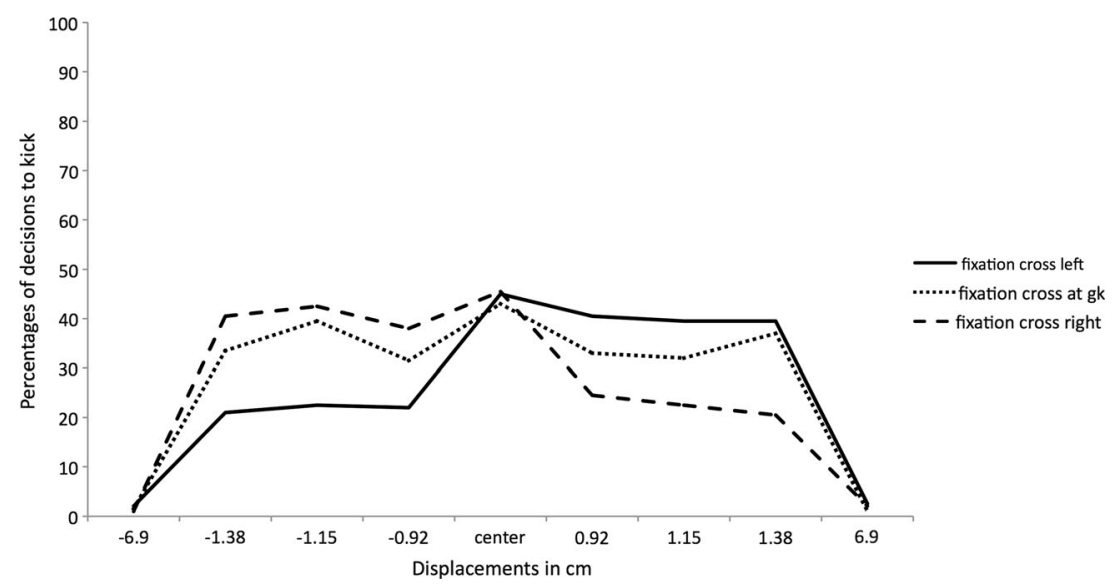

Fig. 1 Percentage of trials in which participants decided to kick as a function of fixation cross location and displacement (from the penalty taker's perspective). Negative values represent displacements to the left side, positive values displacements to the right side 
displacement, $F(8,152)=34.44, p<0.001, \eta_{\mathrm{p}}{ }^{2}=0.64$ and an interaction effect between fixation cross location and displacement $F(16,304)=3.11, p<0.001, \eta_{\mathrm{p}}{ }^{2}=0.14$. The main effect of fixation cross location was not significant, $F(2,38)=0.49$, $p>0.62$. Post hoc analyses indicated that the percentage of trials in which participants decided to kick was larger for the zero and $0.92,1.15$, and $1.38 \mathrm{~cm}$ displacements than for the $6.9 \mathrm{~cm}$ displacements ( $p \mathrm{~s}<0.001$ ). Importantly, participants kicked more often for the small goalkeeper displacements opposite to the side of the fixation cross location (i.e., for a fixation cross at the left post and the goalkeeper displaced to right of the goal center, and vice versa) compared to small goalkeeper displacements to the same side as the fixation location cross $(p s<0.01)$. These differences for fixation cross location did not occur for the zero and $6.9 \mathrm{~cm}$ displacements.

Figure 2 illustrates the percentage of kicks to the side with more space. Notice that for the no displacement category (i.e., with the goalkeeper standing in the exact center), there is no side with more space. In addition, there were almost no kicks for the $6.9 \mathrm{~cm}$ displacements (i.e., the goalkeeper stood obviously off-center). Hence, Fig. 2 depicts the $0.92,1.15$, and $1.38 \mathrm{~cm}$ displacements to the left and right only. Percentage of kicks to the side with more space exceeded $50 \%$, irrespective of fixation cross location. To include as many observations and participants as possible, data for the $0.92,1.15$, and $1.38 \mathrm{~cm}$ displacements were collapsed and submitted to a 3 (fixation cross location: left, right, center) $\times 2$ (direction of displacement: left, right) ANOVA with repeated measures on both factors. This confirmed there were no significant effects of fixation cross location, $F(2,38)=0.2, p>0.82$, displacement, $F(2,38)=0.19 p>0.83$, or their interaction, $F(4,76)=$ $0.63, p>0.64$. Critically, however, participants kicked towards the side with more space more often (i.e., 64.1\%) than they would have done had they picked a side randomly (i.e., $50 \%), t(19)=4.65, p<0.001, d=1.04$. This off-center effect arose for all of the six combinations of fixation cross location

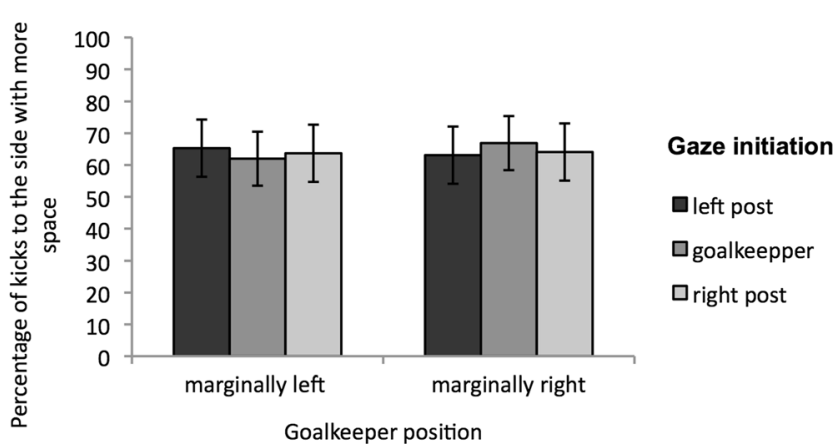

Fig. 2 Percentage of kicks to the side with more space when the goalkeeper was erroneously perceived as standing in the center of the goal, as a function of fixation cross location and displacement (i.e., $0.92,1.15,1.38 \mathrm{~cm}$ to left and right were collapsed into marginal displacements to the left and right; from the penalty taker's perspective). Error bars indicate standard errors and displacement ( $t \mathrm{~s}>2.84, p \mathrm{~s}<0.005, d \mathrm{~s}>0.32$, one-tailed and Bonferroni corrected).

\section{Discussion}

Penalty takers in soccer are inclined to kick to the side of the goal where the goalkeeper leaves marginally more space, even though they are not explicitly aware that the goalkeeper is standing off-center (Masters et al., 2007). To provide further evidence that the off-center effect reflects discrepancies in the accuracy of explicit and implicit perceptions of goalkeeper position, we assessed the influence of scan direction, which systematically affects the explicitly perceived center of lines in landmark discrimination tasks (Varnava et al., 2002), on penalty takers' explicit perception of the goalkeeper's position relative to the goal's center, and asked whether the influence extends to the subsequent selection of the side to which to kick.

Participants initially fixated the right, left or center of the scene. Once the goalkeeper and goal had appeared, the initial fixations typically induced scan patterns across the scene from right to left, left to right, or none. As anticipated, the manipulation of scan direction systematically influenced participants' explicit perception of whether the goalkeeper stood at the exact center of the goal. In agreement with previous work (Varnava et al., 2002), the explicitly perceived center moved slightly away from the side from which participant's started searching the scene. When participants initially fixated the right side of the scene and scanned leftward towards center, then a goalkeeper standing to center's left was more likely to be perceived as positioned in the goal's center (i.e., as reflected by participants taking more kicks) than when the goalkeeper stood to the right of the center, and vice versa for an initial fixation to the left side.

The similarity between effects of scan direction on perception of space in the off-center task and the standard landmark discrimination task (Milner et al., 1992; Varnava et al., 2002) indicates that the two tasks are closely related. However, the bias in perceived midpoint in our task may have been somewhat weaker than typically observed for landmark discrimination tasks. For example, the absence of an asymmetry in spatial perception for initial fixations at the center (i.e., the goalkeeper) may be due to the visual stimulus being in far space. Bias in the landmark discrimination task reduces to zero for lines in far space, rather than shifting to the right as is found for line bisection tasks (Bjoertomt, Cowey \& Walsh, 2002; Cowey, Small, \& Ellis, 1994). Alternatively, the goalkeeper displacements may have been too large, potentially breaching the participants' subliminal threshold for awareness (Dehaene, Changeux, Naccache, Sackur, \& Sergent, 2006). Accordingly, participants actually decided not to kick in a small majority of trials that featured small or no displacements 
(i.e., $66 \%$ ). This suggests that participants were often aware that the goalkeeper stood off-center, even for small displacements.

Importantly, the systematic bias in scan direction was limited to explicit perception of the goalkeeper's position and did not extend to selection of the side to which to kick the ball. That is, participants demonstrated the typical off-center effect of kicking the ball to the side with more space, even though the very fact that they did kick attested to their belief that the goalkeeper actually stood in the true goal center (Masters et al., 2007). To reiterate, the participants' inclination to kick to the side with more space was not influenced by scan direction, because it was equally strong irrespective of displacement and scan direction. We conclude therefore that the effect of scan direction was limited to explicit perception of the goalkeeper position, lending further credence to the conjecture that the off-center effect arises from dissociations between explicit and implicit perception of the goalkeeper's position.

It thus appears that, implicitly, penalty takers have access to more accurate information about the goalkeeper's true position than they do explicitly. This speaks to an ongoing debate in cognitive science about whether implicit perception can really affect behavior (Simons, Hannula, Warren, \& Day, 2006) and whether the evidence is sufficiently rigorous to allow conclusions at all (Newell \& Shanks, 2014). The current findings are not going to resolve this issue, but the differential effects of scan direction do underline the involvement of two separate perceptual processes. Our findings are not sufficient to completely rule out the possibility that the processes are both explicit to some extents, but evidence that the off-center effect occurs (somewhat less pervasively) when participants are encouraged to search for the side with more space (Masters et al., 2007) is consistent with the conjecture that implicit perception affects decision-making behaviour.

One may argue that the two-visual systems theory (Milner \& Goodale, 1995, 2008) offers a more parsimonious explanation of the current findings. The two-visual systems theory holds that separate neuro-anatomical systems exist for perception and action. Deciding whether to kick based on goalkeeper position may involve the ventral perception system, whereas the selection of kick direction may be based on the dorsal action system. However, it is important to emphasize that in the two-visual systems theory, action entails movement control and not action selection (see also van Doorn, van der Kamp, \& Savelsbergh 2007). In particular, recent evidence indicates that aiming in tasks, such as throwing and kicking, strongly relies on the ventral perception stream (Shim et al., 2014). Taxonomies of conscious, preconscious, and subliminal processes (Dehaene et al., 2006) perhaps offer a more fruitful framework for understanding the off-center effect.

\section{Conclusions}

The present findings underline that not all perception of space is infallible and imply that goalkeepers should perhaps be encouraged to try and exploit this to enhance their success. It remains for future research to identify other situations in and outside of sport in which explicit perception of space and subsequent implicit performance diverge.

Acknowledgments This research was supported by a grant from the German Research Council (DFG, Deutsche Forschungsgemeinschaft), ME 2678/14-1, to Daniel Memmert. However, the funding source had no involvement in study design, collection, analysis, interpretation of data, writing this paper, and the decision to submit this article for publication.

\section{References}

Bjoertomt, O., Cowey, A., \& Walsh, V. (2002). Spatial neglect in near and far space investigated by repetitive transcranial magnetic stimulation. Brain, 125, 2012-2022.

Cowey, A., Small, M., \& Ellis, S. (1994). Left visuo-spatial neglect can be worse in far than in near space. Neuropsychologia, 32, 1059-1066.

Dehaene, S., Changeux, J. P., Naccache, L., Sackur, J., \& Sergent, C. (2006). Conscious, preconscious, and subliminal processing: A testable taxonomy. Trends in Cognitive Sciences, 10, 204-211.

Faul, F., Erdfelder, E., Lang, A., \& Buchner, A. (2009). G*Power 3: A flexible statistical power analysis program for the social, behavioral, and biomedical sciences. Behavior Research Methods, 39, 175-191.

Jewell, G., \& McCourt, M. E. (2000). Pseudoneglect: A review and metaanalysis of performance factors in line bisection task. Neuropsychologia, 38, 93-110.

Masters, R. S. W., van der Kamp, J., \& Jackson, R. C. (2007). Imperceptibly off-center goalkeepers influence penalty-kick direction in soccer. Psychological Science, 18, 222-223.

Milner, A. D., Brechmann, M., \& Pagliarini, L. (1992). To halve and not to halve: An analysis of line bisection judgments in normal subjects. Neuropsychologia, 30, 515-526.

Milner, A. D., \& Goodale, M. A. (1995). The visual brain in action. Oxford: Oxford University Press.

Milner, A. D., \& Goodale, M. A. (2008). Two visual systems re-viewed. Neuropsychologia, 46, 774-785.

Newell, B. R., \& Shanks, D. R. (2014). Unconscious influences on decision making: A critical review. Behavioral and Brain Sciences, 37, 1-63.

Nicholls, M. E. R., Loetscher, T., \& Rademacher, M. (2010). Miss to the right: The effect of attentional asymmetries on goal-kicking. PLoS ONE, 5, e12363. doi:10.1371/journal.pone.0012363

Noël, B., Hüttermann, S., van der Kamp, J., \& Memmert, D. (2016). Courting on the beach: How team position implicitly influences decision-making in beach volleyball serves. Journal of Cognitive Psychology.

Noël, B., \& van der Kamp, J. (2012). Gaze behaviour during the soccer penalty kick: An investigation of the effects of strategy and anxiety. International Journal of Sport Psychology, 43, 326-345.

Noël, B., van der Kamp, J., Weigelt, M., \& Memmert, D. (2015). Asymmetries in spatial perception are more prevalent under explicit than implicit attention. Consciousness and Cognition, 34, 10-15. 
Roberts, R., \& Turnbull, O. H. (2010). Putts that get missed on the right: Investigating lateralized attentional biases and the nature of putting errors in golf. Journal of Sports Sciences, 28, 369-374.

Shim, J., van der Kamp, J., Rigby, B. R., Lutz, R., Poolton, J. M., \& Masters, R. S. W. (2014). Taking aim at the Müller-Lyer goalkeeper illusion: An illusion bias in action that originates from the target not being optically specified. Journal of Experimental Psychology: Human Perception and Performance, 40, 1274-1281.

Simons, D. J., Hannula, D. E., Warren, D. E., \& Day, S. E. (2006). Behavioral, neuroimaging, and neuropsychological approaches to implicit perception. In P. Zalazo, M. Moscovitch, \& E. Thompson (Eds.), Cambridge Handbook of Consciousness (pp. 207-250). Ney York: Cambridge University Press.

van der Kamp, J. (2011). Exploring the merits of perceptual anticipation in the soccer penalty kick. Motor Control, 15, 342-358. van Doorn, H., van der Kamp, J., \& Savelsbergh, G. (2007). Grasping the MÃ $1 / 4$ ller-Lyer illusion: The contributions of vision for perception in action. Neuropsychologia, 45, 1939-1947

Varnava, A., McCarthy, M., \& Beaumont, J. G. (2002). Line bisection in normal adults: direction of attentional bias for near and far space. Neuropsychologica, 40, 1372-1378.

Weigelt, M., \& Memmert, D. (2012). Goal-side selection in soccer penalty kicking when viewing natural scenes. Frontiers in Psychology, 3, Article 312.

Weigelt, M., Memmert, D., \& Schack, T. (2012). Kick it like Ballack: The effects of goalkeeping gestures on goal-side selection in experienced soccer players and soccer novices. Journal of Cognitive Psychology, 24, 942-956.

Wood, G., \& Wilson, M. R. (2010). A moving goalkeeper distracts penalty takers and impairs shooting accuracy. Journal of Sport Sciences, 28, 937-946. 